\title{
Les affects et leur destin dans l'intervention
}

Un exemple dans l'industrie automobile

Destiny of affects in the intervention. An example in automotive industry

Jean-Yves Bonnefond et Yves Clot

\section{OpenEdition}

Journals

Édition électronique

URL : http://journals.openedition.org/activites/2895

DOI : 10.4000/activites.2895

ISSN : $1765-2723$

Éditeur

ARPACT - Association Recherches et Pratiques sur les ACTivités

Référence électronique

Jean-Yves Bonnefond et Yves Clot, "Les affects et leur destin dans l'intervention », Activités [En ligne], 13-2 | 2016, mis en ligne le 15 octobre 2016, consulté le 13 novembre 2019. URL : http:// journals.openedition.org/activites/2895 ; DOI : 10.4000/activites.2895

Ce document a été généré automatiquement le 13 novembre 2019.

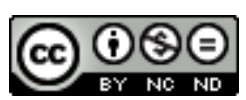

Activités est mis à disposition selon les termes de la licence Creative Commons Attribution - Pas d'Utilisation Commerciale - Pas de Modification 4.0 International. 


\section{Les affects et leur destin dans l'intervention}

Un exemple dans l'industrie automobile

Destiny of affects in the intervention. An example in automotive industry

Jean-Yves Bonnefond et Yves Clot

\section{NOTE DE L'ÉDITEUR}

Article soumis le 22 mars 2016, accepté le 31 juillet 2016

\section{Les mouvements affectifs : objets d'étude et d'action}

1 La question des affects ou des émotions prend de plus en plus de place dans la littérature en psychologie du travail, en ergonomie et, plus largement dans les sciences du travail. Il est sans doute indispensable que la conceptualisation de l'activité s'ouvre sur l'affectivité. On pourrait le penser d'abord au regard de la pratique, tellement l'analyse du travail est confrontée dans les milieux professionnels du monde entier à la montée des formes diversifiées de psychopathologies du travail et on aurait sans doute raison (Almudever, Michaëlis, Aeschlimann, \& Cazals-Ferré, 2011; Clot, 2013; Dejours \& Gernet, 2012 ; Kitanaka, 2014 ; Molinier, 2009; Reille-Baudrin \& Zittoun, 2015 ; Veil, 2012). Mais on se convainc vite que les publications dans ce champ débordent ce domaine. Elles touchent aussi bien à l'étude des émotions en sociologie du travail (Jeantet, 2003) ou en psychologie ergonomique (Cahour \& Lancry, 2011), qu'aux questions de l'intervention ou de la formation professionnelle, dès lors que ces dernières ne négligent pas l'expérience subjective que constitue la réflexion sur l'action proposée aux professionnels dans les organisations (Arnoud \& Falzon, 2013 ; Fernagu-Oudet, 2012). 
2 Succinctement on peut considérer que la sociologie des émotions ou la psychologie ergonomique, au sens large, sont marquées par des lignes de pente distinctes. Une partie de leurs analyses visent à étudier les émotions dans le travail - surtout dans les services - comme un effet prescriptif exposant les sujets à un «travail émotionnel » supplémentaire et couteux pour suivre la prescription. Une autre partie des analyses, sans négliger l'inconfort d'une prescription émotionnelle éprouvante, regardent les émotions comme une source potentielle de transformation. Comme le note, par exemple, Bernard, à l'opposé des risques d'un déterminisme statique, on peut soutenir que l'émotion comporte en elle-même une tendance à l'action $(2015$, p. 8) qui peut faire d'elle " un opérateur de mise en mouvement » (Livet, 2007, p. 340). Dans ce cadre, au-delà d'un objet d'étude, elle peut devenir un moyen de transformation de l'activité. C'est le cas, par exemple, quand des interventions en ergonomie, sans instruire la question des émotions ou des affects en tant que tels, rencontrent ces mouvements affectifs : ainsi, dans la méthode de "co-analyse constructive des pratiques", consistant à mettre en débat le travail entre différents professionnels observant le travail de leurs collègues, «l'opérateur [...] placé au cœur de la situation de son partenaire, peut rebondir sur les doutes quotidiens ou faire l'expérience de la "surprise" à travers l'observation. » (Arnoud \& Falzon, 2013, p. 231). Ces «surprises» indiquent selon les auteurs que «la situation observée ne correspond pas aux attentes ou aux représentations de l'opérateur » qui observe son collègue en train de travailler (Ibid.). Émotions et affects ne sont certes pas mentionnés ici, mais ils sont pourtant indubitablement présents dans la «surprise » identifiée chez les opérateurs.

3 Ils sont par contre explicitement mentionnés dans une partie de la littérature de psychologie ergonomique où ils peuvent alors se trouver étudiés en tant que tels mais pas d'abord pour leur motricité dans l'intervention. Ils sont de nouveau perçus alors comme des perturbateurs potentiels. Même si leur rôle de moyens nécessaires et potentiellement bénéfiques à la réalisation du travail est souligné dans ces études, ce "travail émotionnel » (terme emprunté aux travaux de Hochschild, 2003) mobilisé par le sujet est coûteux en termes de charge de travail et son évaluation devient essentielle "pour contribuer à faire diminuer la charge et améliorer les conditions de travail » (Ribert-Van de Weerdt, 2011, p. 322). L'objet de l'intervention consiste donc à repérer les sources d'émotions dans le travail pour limiter leurs aspects négatifs sur la santé et favoriser leurs aspects positifs. L'application de stratégies de "gestion de l'émotion » par le sujet est vue comme un coût qui peut devenir néfaste pour la santé par une mobilisation trop importante de ressources qui « crée de la fatigue, voire de l'épuisement » (Ibid., p. 334).

Objet d'étude dans ce cas, les états affectifs concernent l'activité ici conçue classiquement comme «ce qui est réellement mis en œuvre par un sujet dans une situation donnée » (Cahour, 2010, p. 274) et que la tâche rend «couteuse». Mais, quand on cherche à transformer l'activité et pas seulement à la connaître, quelle fonction occupent ces états affectifs? Comment "comprendre ce que "font" les situations de confrontation aux traces de sa propre activité » (Cahour \& Licoppe, 2010, p. 244). Et surtout, quel est le rôle de l'intervenant-chercheur dans ces processus affectifs surprenants, sources d'éventuelles transformations, mais aussi d'inhibitions possibles?

5 Les émotions, si l'on suit de récents travaux en psychologie ergonomique, ne doivent plus être « considérées comme un facteur marginal intervenant de temps en temps, mais bien comme constitutif de toute activité qui se déploie dans un flot constant et mouvant d'états émotionnels qui orientent et impactent l'action, la relation et la réflexion, et qui 
sont eux-mêmes modifiés par l'activité qui se déploie et la situation qui se construit. » (Cahour \& Lancry, 2011, p. 105).

6 Mais alors il faut sûrement prendre au sérieux une conception développementale de ces émotions et de l'activité qui, loin de pouvoir se réduire à ce qui se fait dans la situation présente, se conjugue aussi au futur, comme chez Vygotski : « l'homme est plein à chaque minute de possibilités non réalisées " (2003, p. 76). C'est d'autant plus vrai en cours d'intervention. C'est pourquoi l'objectif de cet article est de regarder les mouvements affectifs comme le ressort même de l'action de transformation.

On voudrait montrer que l'affectivité se développe aussi en cours d'activité. Les rapports entre activité et affectivité sont subtils, mais les résultats obtenus par Vygotski montrent qu'on ne peut pas faire de l'affectivité une valeur autochtone ou originaire. Il faut la voir en mouvement dans l'activité. Il n'est pas facile en psychologie d'assumer jusqu'au bout le paradigme de l'activité; pas facile d'éviter le dualisme entre d'un côté, une activité réduite à ce qu'on fait et, de l'autre, une subjectivité autochtone trouvant ses ressorts en elle-même. C'est pourtant ce dualisme qui expose la psychologie du travail et l'ergonomie soit à s'occuper du sujet sans vraiment s'occuper du travail (Clot, 2010; Clot \& Simonet, 2015) soit à s'occuper du travail sans véritablement s'occuper du sujet (Van Bellegen, De Gasparo, \& Gaillard, 2013).

Bien sûr, une clinique de l'activité est aussi régulièrement confrontée à ces processus psychiques au cours des activités dialogiques provoquées dans la variété des cadres méthodologiques mis en œuvre (Clot, 2008; Fernandez, 2009; Kostulski, 2012 ; Roger, 2007 ; Scheller, 2013). Dans les interventions pour transformer le travail, l'ampleur des manifestations affectives rencontrées soulève des difficultés cliniques importantes. Cet article, à partir d'une analyse de cas, cherche à contribuer à la réflexion théorique en la matière. En effet, l'action pour étendre le pouvoir d'agir des professionnels met «en activité » l'affectivité de ces derniers. Dès lors, sans prise en compte clinique des obstacles qu'elle soulève et des ressources qu'elle recèle, la passivité risque de l'emporter sur le développement de l'activité. C'est d'autant plus le cas dans les situations de travail très dégradées aujourd'hui, où l'on rencontre beaucoup d'affects tristes au cœur d'une activité empêchée qui a souvent " mal tournée », qui vire au ressentiment, nourri par l'amertume devant les efforts gâchés et la parole inutile. C'est avec ce que nous appellerons plus bas des « affects passifs » qu'il nous faut travailler souvent pour trouver un passage vers des "affects actifs" aptes à recharger en énergie l'activité collective et individuelle. L'affectivité en cours d'activité se présentant donc d'abord comme un risque pour le développement de l'activité, on vise à en faire une ressource aussi bien dans le cadre des méthodes d'analyse comme les auto-confrontations (Bonnemain, 2015; Bonnemain \& Clot, à paraître; Clot, 2008; Clot, Faïta, Fernandez, \& Scheller, 2001; Duboscq \& Clot, 2010) que dans l'ensemble de l'intervention (Bonnefond, 2015).

\section{La différence entre affect et émotion}

9 Dans cet article, après d'autres travaux (Bonnemain, 2015; Clot, 2015b; 2016; Poussin, 2014) on prend le parti d'une distinction conceptuelle entre affect et émotion insuffisamment faite dans la littérature où le flottement dans la définition de leurs différences est souvent un obstacle (Rimé, 2009). C'est pourtant important, du point de vue développemental, c'est-à-dire du point de vue de l'action, de les différencier. L'émotion signale incontestablement l'affect, le rend plus ou moins visible et observable, 
mais il ne s'identifie pas avec elle. C'est chez Wallon qu'on trouve la définition la plus claire des émotions (Bautier \& Rochex, 1999; Rochex, 2002). Pour lui, elles sont, individuellement et collectivement la manifestation d'une excitation et même d'un « tumulte » organique. Toutes les émotions qui extériorisent plaisir, joie, colère, angoisse, peur, timidité, « peuvent être ramenées à la manière dont le tonus se forme, se consomme ou se conserve » (Wallon, 1982, p. 208). C'est un état effervescent du corps et de l'esprit, brusque, disruptif et épisodique. Elles trahissent l'affect, aux deux sens du terme. Elles le révèlent, mais n'en fixent pas directement la teneur. Sans pouvoir insister plus longuement sur cette différence dans le cadre de cet article (Clot, à paraître ; Poussin \& Miossec, 2015) on peut proposer pour spécifier ici les affects, une définition inspirée par Livet qui nous permet de les distinguer des émotions, au moins pour l'analyse alors même qu'ils sont, bien sûr, organiquement liés dans l'action réelle : devant le différentiel entre nos attentes en cours et les traits d'une situation qui surgissent, dans le conflit qui se forme donc entre nos attendus et les inattendus de l'activité en cours, l'émotion n'est que le signe extérieur de l'affect, elle ne constitue qu'une "sonnette d'alarme pour nos révisions. » (Livet, 2007, p. 340).

C'est ce travail de "révision " nécessaire qui nous affecte en faisant osciller les rapports de forces dans la marche du connu vers l'inconnu. De ce point de vue, l'affect est bien plus qu'un ressenti subjectif. C'est une force de travail (Green, 2004, p. 100). Les destins de l'action sont envisagés par Livet selon deux grandes modalités de réalisation possibles en balance. Le travail affectif de révision nécessite de mettre sa perception du monde en accord avec ce que nous dit le monde réel. Dans un premier cas, si «la nouvelle information du monde est "une bonne surprise", la révision est facile et réalisée avec plaisir» (Livet, 2002, p. 125). Dans un second cas au contraire, "lorsqu'il s'agit de perceptions plus négatives [...] le travail de révision nécessite d'aller contre des préférences profondément ancrées, dont certaines sont étayées sur des valeurs importantes pour le sujet. » (ibid.). On dira alors que les affects peuvent déboucher sur un «blocage des révisions» (Livet, 2007, p. 341), en empêchant la transformation de la perception et des attentes. On peut ajouter que ce «blocage » de l'affect peut se réaliser sous des modalités émotionnelles diversifiées qu'on ne peut confondre avec lui. L'affect, on va le voir, est un devenir de l'activité qui met en balance les attendus et les inattendus, une exigence de travail qui libère de l'énergie disponible pour l'action sans garantir d'avance ni sa réalisation, ni la direction que prendra cette action.

Dans cette optique, nous cherchons ici à discuter le rapport entre l'affect et l'activité pratique dans la perspective développementale des interventions en psychologie du travail sous les modalités de la clinique de l'activité. Nous mobilisons une conception de l'affect défini comme un conflit de l'activité avec elle-même, épreuve subjective dans laquelle le devenir imprédictible de l'affect balance entre deux pôles, actif et passif ou, plus justement, hésite entre deux orientations, passive et active, entre deux "allures " prises par l'activité (Canguilhem, 1984, p. 137). Pour cela, on s'intéressera donc aux actes effectifs réalisés dans le cadre clinique, en interrogeant leur portée transformatrice ou au contraire défensive. Nous verrons que le cadre clinique dialogique est la source du développement possible de l'affect pour un devenir actif, s'il est le moyen d'une « révision » au sens défini ci-dessus : pour qu'un problème analysé sans avoir été d'abord résolu, soit, non pas refoulé dans l'organisation, mais, au contraire objet d'une repriserépétition dans un autre contexte où sa répétition est non seulement admise, mais sollicitée. C'est ce que nous exposerons à partir d'un exemple très circonscrit, tiré d'une 
intervention dans un groupe industriel, chez un constructeur automobile. Mais il nous faut, auparavant, mieux définir ce que nous entendons par affect dans l'activité.

\section{L'activité : deux conflits régénérés par l'intervention} que nous commencerons ici par identifier, mais dont on trouve déjà une première caractérisation ailleurs (Bonnemain, 2015 ; Clot, 2015a ; 2016 ; Poussin, 2014).

Le premier conflit est inhérent au caractère triplement dirigé de l'activité qui fait d'elle une «triade vivante", la plus petite unité de l'échange social (Clot, 1999; 2008; 2016 ; Fernandez, 2009 ; Kloetzer, Clot, \& Quillerou-Grivot, 2015). En effet, l'activité n'est jamais celle d'un sujet sans destinataire. Elle est adressée. Elle n'est pas seulement dirigée vers son objet, elle est dirigée simultanément vers l'activité des autres qui porte sur le même objet et vers les autres activités possibles du sujet. Les discordances entre ces pôles rendent par définition conflictuelle l'activité en cours, ce qui d'ailleurs rend possible son développement, car sans conflit l'activité reste sans possibilité. Le rapport d'un sujet à l'objet de son activité n'existe pas sans le rapport à autrui sur cet objet, de même que les relations à autrui ne sont pas indépendantes du rapport à l'objet. Cet objet, pris dans les rapports sociaux est donc aussi l'objet d'autres activités, où chacun a ses buts assignés et ceux qu'il se fixe. De fait, il y a une sorte de collision dans l'objet où ces différents buts à atteindre en rivalité éventuelle sont à l'origine du conflit de critères sur la qualité du travail. C'est ce qui en fait la source inépuisable d'une régénération, au moins potentielle, du rapport social aux objets du monde (Clot, 2016). On le sait, la vitalité de ce conflit normal dans l'activité à propos de l'objet du travail, qui débouche in fine sur des délibérations potentielles autour des critères du travail bien fait, est en réalité largement malmenée dans les organisations contemporaines et ce jusque dans l'activité la plus personnelle (Lhuilier, 2014). En effet, faute d'institutions dialogiques, ce conflit moteur se retourne souvent contre la santé et l'efficacité (Clot, 2010; Clot \& Gollac, 2014, Tomàs \& Bonnefond, 2014). C'est ce conflit de critères que restaure, dans le cadre clinique, l'organisation du dialogue sur le travail où se renouvelle l'activité réflexive sur ce dernier. En renouvelant les objets de discussions entre professionnels dans le collectif, puis entre eux et leur hiérarchie sur ces mêmes objets, on engage les protagonistes de l'organisation à éprouver les attendus habituels de leur expérience individuelle et collective.

Ces attendus de l'expérience, on le sait, équipe les professionnels d'instruments génériques qui donnent un répondant collectif à leur activité individuelle. Il s'agit des expériences déjà faites consolidées en perceptions présupposées, en attentes préméditées, en raccourcis opératoires partagés à plusieurs qui sont comme des mots de passe connus seulement de ceux qui appartiennent au même horizon de travail. On a appelé genre professionnel ces manières de dire ou de prendre habituellement les choses et les gens qui pré-organisent l'activité personnelle grâce à des évaluations communes qui sont autant d'instruments réglant cette activité personnelle de façon tacite (Clot \& Faiita, 2000).

Sous l'impact du premier conflit de l'activité adressée, qui est comme le trait d'union entre les objets, le sujet et autrui - conflit le plus souvent régénéré par l'intervention un deuxième conflit marque l'épreuve affective que traverse l'activité en cours, lorsque les instruments génériques prêts à servir sont " pris en défaut ", soumis à une " révision " plus ou moins brutale dans le cadre dialogique de l'intervention. Ce cadre constitue à ce 
titre une épreuve affective où, pour le dire cette fois à la manière de G. Canguilhem, «l'homme ne peut pas se dérober devant les problèmes que lui posent les bouleversements parfois subits de ses habitudes » (1984, p. 133). Et là aussi, les opérateurs mesurent leur santé à leur capacité de surmonter ces habitudes, bonnes ou mauvaises, pour en expérimenter de nouvelles. Il ajoute: "par vie on peut entendre le participe présent ou le participe passé du verbe vivre, le vivant et le vécu. La deuxième acception est selon moi, commandée par la première qui est plus fondamentale» (1983, p. 335). Pour lui, c'est donc bien l'activité en cours qui parvient ou non à mobiliser l'expérience déjà vécue en moyen d'en vivre une autre. L'affect est donc un conflit plus ou moins intense (Bonnemain, 2015) entre l'expérience qu'on a et l'expérience qu'on fait (Pastré, 2012, p. 120), conflit où se régénère ou dégénère l'activité. On a ci-dessus marqué l'importance de cette "révision" nécessaire de l'expérience lorsque la situation ne se présente pas aux sujets comme prévue. Et on peut considérer que toute intervention confronte les demandeurs à cette question.

\section{Affects passifs et actifs : se relier au réel}

On insistera maintenant sur les deux destins de cette « révision ». Le premier d'entre eux que nous avons défini plus haut comme un «blocage de l'affect " peut être décrit comme une défense plus ou moins anxieuse des habitudes alors que le deuxième relève de ce que Vygotski identifiait justement comme un développement: lorsque l'expérience vécue peut changer de statut et quelle devient un moyen de vivre de nouvelles expériences. Avec toutes les intermittences qui s'y attachent, on peut donc dire que l'affect vital fluctue toujours nécessairement entre passivité et activité lorsque les sujets sont à l'épreuve de la révision de leur expérience. Mais l'affect - cette balance de la passivité et de l'activité et même cette indistinction transitoire entre les deux - sera dit ici plutôt actif quand le sujet parvient à faire du déjà vécu un moyen de vivre autre chose ; il sera dit plutôt passif quand, pour lui, l'activité vivante en cours n'est plus, au contraire, que le moyen de vivre toujours la même chose (Clot, 2016). Quand l'épreuve de l'expérience déroutante n'est plus, pour les sujets concernés, que la preuve supplémentaire que les choses seront toujours comme elles sont, on peut dire que le destin passif de l'affect l'emporte. Ce dernier interrompt, à l'insu des sujets le plus souvent, la marche du connu à l'inconnu pourtant impliquée dans l'engagement de l'analyse de sa propre activité proposée par l'intervention. On peut penser que la passivité l'emporte dans l'affect lorsque les sujets rabattent l'imagination de solutions auxquelles personne n'a encore pensé sur la répétition à l'identique du déjà fait, déjà dit, déjà pensé, ensemble ou individuellement.

17 Il faut pourtant insister sur la fragilité et la subtilité de ces passages de la passivité à l'activité et réciproquement. Une tendance supposant l'autre, l'enjeu pour l'intervenant est plutôt de tirer le meilleur parti clinico-développemental de cette variabilité. On pense ici à la remarque prudente de P. Macherey en commentaire de Spinoza qui a, le premier, fait cette distinction entre affect passif et actif :

«Le caractère essentiel de la vie affective étant son instabilité, puisqu'elle se déploie entre un pôle passif et un pôle actif qui la tirent tantôt dans le sens du pire et tantôt dans le sens du mieux, une éthique raisonnée se fixe pour programme de tirer le meilleur parti possible de cette variabilité, en cherchant à exploiter les "mauvais" affects dans le sens du bien qu'ils peuvent comporter, et à limiter le mal qui demeure attaché au fonctionnement des "bons" affects, dont il faut savoir qu'ils 
ne sont jamais définitivement protégés contre les risques de dérive qui en

contrarient les manifestations » $(1999$, p. 113).

On peut comprendre du coup, pourquoi il paraît préférable d'écarter un vocabulaire moraliste qui opposerait des affects positifs à des affects négatifs. On verra justement cidessous à quel point passivité et activité peuvent s'inverser, combien l'une peut prendre le visage de l'autre et même apparaître pour ce qu'elle n'est pas, dès lors que l'affectivité est mise au travail dans l'intervention et que son développement est recherché pour développer l'action des professionnels concernés. C'est l'objet de cet article. Il nous permettra de corroborer les résultats obtenus par A. Phillips dans un autre domaine :

«chaque fois que nous sommes capables de renoncer à une conviction qui s'est

révélée fausse [...] nous nous relions au monde » (2005, p. 156).

L'intervention telle que nous la concevons (Bonnefond, 2015; Briec, 2013; Clot, 2014 ; Kostulski, Clot, Litim, \& Plateau, 2011; Litim, 2012 ; Miossec, Clot, \& Boucher, 2014 ; Prot, 2006) cherche en quelque sorte à opérer, non sans difficulté, cette reliaison au réel du travail. Et cette reliaison concerne chacun des quatre registres du métier identifiés en clinique de l'activité : aussi bien l'activité personnelle que l'activité interpersonnelle des opérateurs réunis en collectifs de travail et aux prises avec l'histoire transpersonnelle et générique de ces collectifs. Mais elle concerne aussi, bien sûr, les hiérarchies et la Direction de l'organisation qui définit les prescriptions impersonnelles organisant les activités des opérateurs. Mieux, ce travail de reliaison touche aux rapports entre ces registres différents dont la discordance plus ou moins créatrice assure la dynamique des métiers concernés au contact du réel. L'intervention à laquelle nous empruntons l'exemple qui suit s'efforce, sur chacun des registres et entre eux, d'exercer, chez les professionnels, cette capacité à surmonter les habitudes qui font obstacle au développement de l'activité. La méthodologie développementale qu'elle mobilise, aussi bien dans le cadre réglé des autoconfrontations qu'au sein des comités de pilotage euxmêmes (Quillerou-Grivot \& Clot, 2014), cherche donc à instituer un mouvement affectif entre le déjà fait, le déjà dit, déjà pensé et le pas encore fait, dit ou pensé. Et ce, en tirant le meilleur parti, dans l'action, de la variabilité constitutive des affects décrite plus haut; afin que les sujets puissent développer leur pouvoir d'agir sur eux-mêmes et sur leur milieu, jusqu'à modifier ce qui peut l'être dans l'organisation du travail pour qu'elle "descende » au plus près du réel. La tâche, dans ce qu'elle a nécessairement de plus impersonnel fixe, le plus souvent, les compromis passés entre les concepteurs et les managers à propos des représentations qu'ils se font du réel et des opérateurs. Ce « déjà pensé » mérite, on va le voir, les « révisions » qui s'imposent.

\section{L'intervention à l'usine Renault de Flins : dispositif clinique}

19 À l'origine de l'expérimentation réalisée avec Renault au sein de l'usine de Flins, il y a une demande de la direction de l'entreprise d'instruire des désaccords qui sont en impasse entre direction et syndicats sur l'appréciation des situations de travail en matière de santé et de qualité. Notre proposition, acceptée par tous, fut non pas d'arbitrer ces désaccords, mais d'expérimenter à plusieurs niveaux dans l'entreprise des moyens de dialogue sur la qualité du travail, conflictuelle par nature, pour construire des arbitrages organisationnels nouveaux qui soient source de santé et de performance. Il fut convenu 
d'expérimenter cela en usine puis dans l'ingénierie. Notre propos ici ne porte que sur l'usine de Flins. celui de l'atelier avec une UET (Unité Elémentaire de Travail) d'habillage des portes. Ensuite, celui de l'usine avec un comité local réunissant les organisations syndicales locales, mais aussi centrales, la direction usine et la ligne hiérarchique, la DRH usine et entreprise, le service santé au travail, les intervenants Cnam, et le moment venu les opérateurs impliqués. En effet, la présence volontaire de ces derniers est consécutive à leur engagement dans le dialogue entre eux sur la qualité de leur travail au niveau de l'UET. C'est durant cette phase préalable que se construisent les conditions de leur contribution au dialogue sur le travail réel avec la hiérarchie et les syndicats en comité de suivi. des portes afin de construire avec ceux qui le demandent, une analyse par eux avec nous de leur activité préalablement filmée. Nous mobilisons pour cela la méthode des autoconfrontations croisées (Clot, 2008; Clot, et al. 2001) ; c'est le temps de la dispute professionnelle entre connaisseurs que sont les professionnels concernés, sans participation de la hiérarchie. C'est le temps du travail collectif autour des plaisirs et des déplaisirs de la controverse sur le travail bien fait, qui vise à développer la fonction psychologique du collectif pour imaginer de nouvelles possibilités de penser et d'agir. Ensuite, les résultats produits (sous forme de montage vidéo) font l'objet d'un autre dialogue au sein des comités de suivi. Ainsi, le collectif change de place dans le cours de l'intervention en devenant ressource pour modifier si possible l'organisation du travail. L'ensemble de ces processus dialogiques dans le cadre général d'intervention est à l'origine de mouvements affectifs puissants, en autoconfrontation d'abord (Bonnemain, 2015), et dans les comités de suivi qui font l'objet d'un travail clinique particulier (Quillerou-Grivot \& Clot, 2014). Nous l'avons déjà évoqué, l'impact premier de l'affect est de désorganiser l'activité habituelle du sujet, qui s'intensifie sous le coup de la destitution de ce qui était tenu pour acquis, que ce soit du côté de l'objet, des destinataires, ou des instruments. Ce changement de rythme de l'activité ouvre alors potentiellement sur quelque chose de nouveau, sur un développement de l'activité, mais aussi sur le risque d'une défense plus ou moins anxieuse des habitudes. Dans ce devenir imprédictible de l'affect, l'activité clinique est convoquée pour soutenir et accompagner, sans garantie d'avance, un devenir actif.

Bonnemain (2015), lors d'une recherche auprès de chefs d'équipe de la propreté de Paris a étudié, dans le cadre clinique des autoconfrontations croisées, la fonction de l'affect et son issue développementale dans l'activité dialogique. Il montre comment dans les faits, non seulement le rapport actif/passif de l'affect est très fluctuant, mais également que l'affect est d'abord nécessairement passif : « Nos résultats nous permettent de soutenir que l'intensité affective de l'activité n'est jamais totalement tournée vers l'activité ou vers la passivité. On constate plutôt des formes d'alternances systématiques entre les deux dans l'activité du sujet. On constate même dans chacune des séquences que cette intensité est d'abord passive: l'affect semble d'abord nécessairement passif face à un inattendu qui surgit dans l'activité du sujet. » (p. 284). Bonnemain note que ce moment nécessairement passif de contact avec l'imprévu rend possible, paradoxalement, un 
développement de l'activité dans l'échange entre professionnels et avec le clinicien, mais à une condition : que le dialogue permette une "prise en charge » de ces affects passifs par leur transfert entre les participants. Autrement dit, que l'imprévu à la source du décontenancement $d u$ sujet fasse l'objet de reprises par les participants pour son élaboration. Ce processus rend alors possible la poursuite du dialogue intérieur et extérieur au principe du développement de la pensée (Kostulski, 2011; Kostulski \& Clot 2007 ; Scheller, 2013; Vygotski, 1997). En effet, la passivité initiale dans la situation inattendue s'avère nécessaire, elle permet aux sujets d'abord de supporter l'inconnu. Mais elle n'est pas suffisante: quand il existe, "C'est l'échange qui conduit ensuite cette passivité vers les formes actives de son développement, dans un jeu affectif entre les différents protagonistes du dialogue. » (Bonnemain 2015, p. 284). Etant entendu que l'intervention vise le développement du pouvoir d'agir des professionnels sur leur travail, voyons comment, à partir du développement d'une situation concrète, appréhender le devenir de l'affect dans des actes effectifs. Entre les affects actifs et le développement réalisé de l'activité, entre eux et elle, il y a un pas : celui de la mutation tangible des objets, des destinataires et des instruments de l'action du sujet. C'est seulement au vu de ces transformations qu'on dira les affects finalement actifs. Non pas seulement sur le seul critère de l'énergie déployée, mais sur celui du développement effectif de l'activité contre la passivité de l'organisation impersonnelle du travail.

Avec ce que nous avons précisé jusqu'ici, nous voudrions montrer que ce chemin vers une issue « finalement active » n'a rien de linéaire. Il passe par des bifurcations dialogiques. Mais on y rencontre aussi des actes pratiques que l'on pourra qualifier de passifs et dont il faut pouvoir tirer pourtant le meilleur parti pour le développement de l'activité.

\section{Un problème de « lumière au poste » : le temps de l'affect}

Dans l'unité (UET) d'habillage des portes où a débuté l'expérimentation, deux équipes se relaient pour couvrir les seize heures de production quotidienne. Dans ces deux équipes, vingt opérateurs et opératrices se sont engagés dans les autoconfrontations croisées portant sur dix postes, donnant lieu à un montage vidéo, adressé au premier comité de suivi. Ces dialogues filmés sur le métier, véritables diagnostics d'experts sur la qualité du travail réel, sont des moyens à la fois technique et affectif, qui posent les bases des échanges en comité de suivi. Ainsi ont été donnés à voir et à entendre : l'engagement des opérateurs dans leur travail, la qualité de leurs analyses, leur ingéniosité, mais aussi les compensations parfois au prix de leur santé, des problèmes de conception de pièces, de moyens, d'organisation $\mathrm{du}$ poste. De surcroit apparaissait un refoulement organisationnel, ce que l'on a qualifié de "parole inutile », c'est-à-dire, l'expérience répétée d'avoir parlé, signalé, proposé, sans que cela puisse porter. Cette expérience de l'inutilité de la parole s'est transformée au plan psychologique en sentiment partagé (Poussin, 2015) qu'il est vain de dire que rien ne peut changer.

2). 
Figure 1 : Déplacement anormal.

Figure 1: Abnormal movement

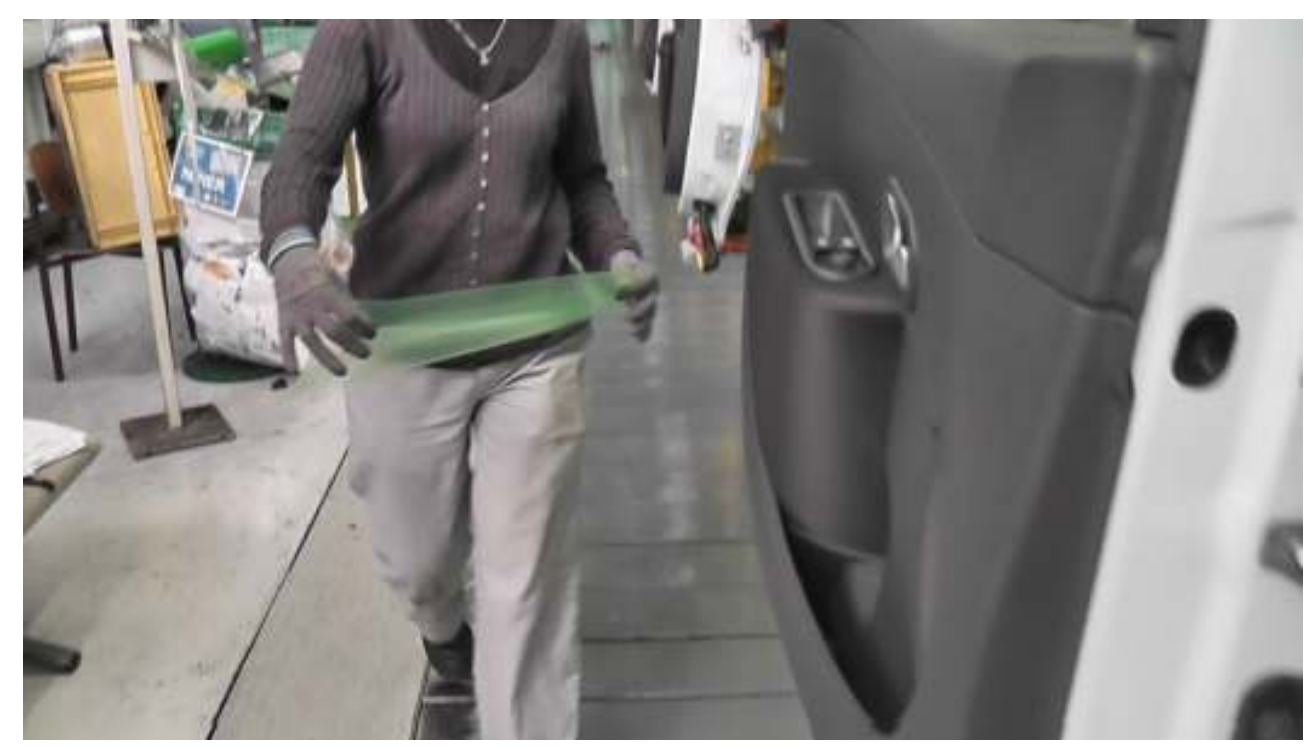

Figure 2 : Affect et dialogue sur ce déplacement. Figure 2: Affect and dialogue about this movement

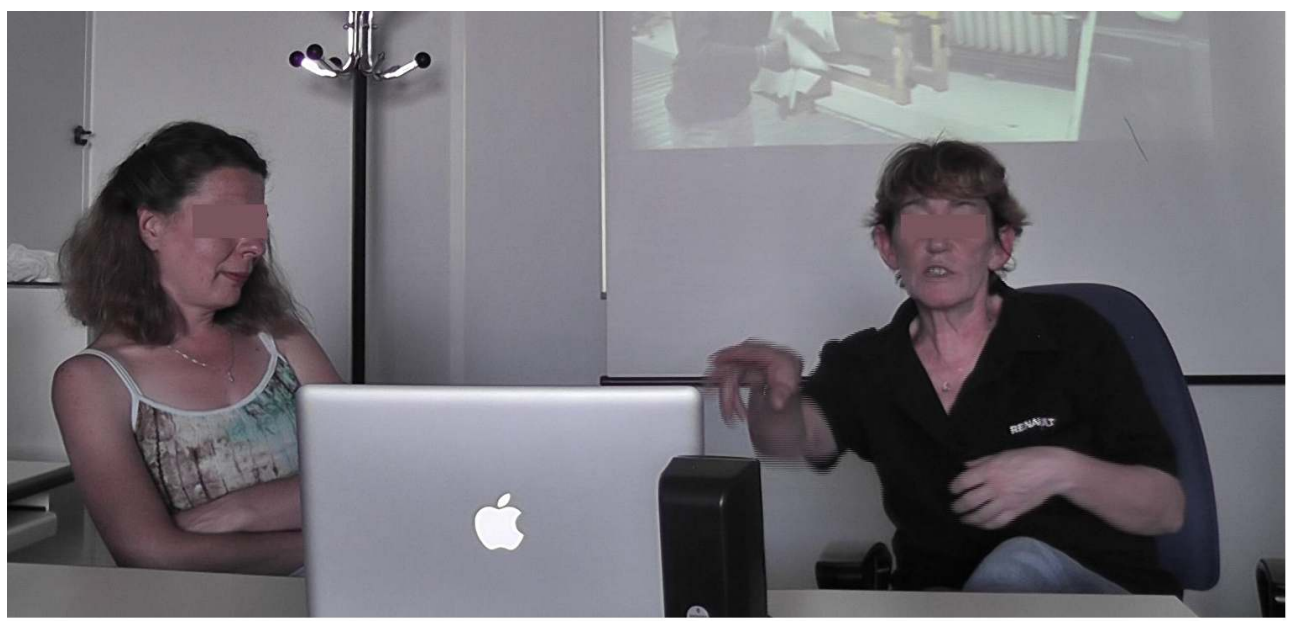

Il s'agit du poste dit de "Checkman », qui dans chacune des deux équipes est tenu par une opératrice. C'est le dernier poste de la ligne des portes, l'opératrice est chargée de « verrouiller » la qualité, de faire en sorte qu'aucun défaut ne sorte de l'UET, elle doit donc les détecter pour qu'ils soient corrigés par un retoucheur de l'équipe ou enregistrés et traités plus loin s'ils sont non retouchables ici. Dans l'autoconfrontation croisée (Figure 2), l'opératrice redécouvre alors avec sa collègue, à la vue des images de son activité, son propre renoncement tacite, dans ce cas précis, à parler, avec sa hiérarchie des obstacles récurrents dans son activité, de surplus, contraire à la performance et à la qualité. Elle se voit faire plusieurs pas supplémentaires pour remonter vers un distributeur de films plastiques protecteurs, prendre un film, revenir où elle travaille (Figure 1), puis le coller sur le bas du panneau de chaque porte. Elle réalise alors non sans surprise combien elle travaille en aval de l'emplacement prévu pour son poste où l'éclairage est défaillant. Son étonnement signe l'affect. 
Op1 : Normalement je devrais mettre les films juste en face de ma table, mais c'est normal, c'est parce que je veux de la lumière, à ma table j'ai pas de lumière, on n'a pas d'éclairage

Op2 : Non l'éclairage n'est pas bon, il y a un coin c'est vraiment sombre

Psy : Et ça il faut faire avec? Il n'y a pas eu de tentative.

Op1: On a tenté, mais ça n'a pas marché.

Op 2 : On a tenté, mais on va couter beaucoup trop cher. ou pourrait être, tel, en tous cas, qu'il se présente dans les films d'autoconfrontation analysés ensemble. Cette mise en visibilité des handicaps organisationnels, compensés par l'expertise des professionnels et leur activité permanente de récupération (Faverge, 1979) est une épreuve dans le cadre clinique du comité de suivi et provoque une sorte de « révision » nécessaire de l'organisation existante à ce moment-là destituée. Or, l'impersonnel, l'organisation dans la situation du Comité, c'est l'activité personnelle incarnée du ou des dirigeants et responsables hiérarchiques en présence de la trace filmique de l'activité au poste et de l'activité dialogique des opératrices sur cette même trace. Ici l'intensité des affects éprouvés par le dirigeant ou les dirigeants et leur devenir actif/passif sont un enjeu clinique central.

technique, le problème de lumière est un " petit » problème à l'échelle de l'usine alors même qu'il a des conséquences lourdes sur la qualité finale du véhicule. Cet écart même suscite une tension qui potentialise une réaction concrète. Et ce, d'autant plus que nombre des problèmes présents dans le montage vidéo, exposés, discutés en comité de suivi ne sont pas aussi simples que celui-ci et témoignent d'une certaine impuissance 
organisationnelle. Dans ces conditions, les affects afférents à l'embarras provoqué par le montage vidéo chez des dirigeants "décontenancés » par la situation vont provoquer le traitement accéléré du problème technique de l'éclairage à ce poste. Toute la ligne hiérarchique, depuis le chef d'atelier jusqu'au directeur d'usine va s'empresser de faire disparaître ce problème de lumière et s'assurer qu'il a bien disparu définitivement. Que penser de ces actes? Les affects éprouvés par la ligne hiérarchique et leur suite concrète sont-ils « actifs ", au sens défini plus haut?

Soyons nets: la lumière convenable restaurée au poste est un résultat tangible et ce retour «à la normale » grâce à cette transformation du travail est plus que louable. À commencer par les opératrices, tous les protagonistes vont trouver légitime que l'on «traite» le problème. Cependant le traitement pratique immédiat de ce problème technique, parmi tant d'autres, laisse en l'état une toute autre question: le statut de la " parole inutile » à ce point chronique dans l'organisation, qu'un problème aussi simple à régler que celui-là peut se trouver durablement refoulé. Le règlement technique du problème de la "lumière» ne lève aucunement ce refoulement. Au contraire la précipitation mise à le faire disparaître du dialogue dans le dispositif d'intervention devient un symptôme du refoulement du dialogue lui-même. Les opérateurs, dans le cadre clinique proposé, ont réalisé - non sans s'étonner du coup de leur propre posture - à quel point eux-mêmes avaient renoncé à instruire ce problème simple avec leur hiérarchie. C'est la source organisationnelle de ce renoncement dialogique que l'intervention réactualise.

Pour les opérateurs, la «lumière » abandonnée à l'anonymat de l'organisation pose le problème $\mathrm{du}$ silence hiérarchique devant le réel du travail; et de leur propre «contamination» par ce silence. Mais la précipitation opérationnelle de la hiérarchie - au demeurant si précieuse pour la santé et la qualité à court terme - reconduit simultanément ce silence. La lumière, si l'on peut dire, doit être rétablie au plus vite pour éviter que lumière soit faite sur la défaillance dialogique chronique de l'organisation. Le rétablissement de la lumière éteint à nouveau le dialogue. En cela, on peut considérer que ce rétablissement concret, qui résout pourtant un problème important est aussi du côté de l'affect passif, du blocage de l'affect mentionné plus haut, celui qui reconduit le déjà vécu à la répétition. Le "réflexe managérial » répète là, en effet, les schèmes consolidés lors de visites d'usine ou d'audits divers et variés qui multiplient les corrections souvent factices et pour le moins précipitées du process. Sur ce registre spécifique, rien de nouveau ne se produit ici, le déjà vécu s'actualise sans régénération de l'activité : cette activité rabat les surprises de la situation sur le fonctionnement organisationnel existant, à savoir sur une répétition de la "mauvaise " habitude organisationnelle consistant à colmater par des solutions d'urgence le déni d'un conflit de critères sans institution dialogique. Une "passivité joyeuse» (Séverac, 2005, p. 22) est au rendez-vous de cet activisme. S'y manifeste la puissante vitalité d'un affect confisqué par des habitudes nécrosées qui déterminent une « révision » faussée.

Pourtant cette passivité ne doit pas être prise à la lettre. Cette répétition n'est pas vouée à la répétition à l'identique. Son destin n'est pas écrit d'avance.

\section{Répétition : I'habitude en question, temps deux}

37 La répétition de ce qu'il faut bien appeler un symptôme organisationnel est peut-être nécessaire dans le dispositif d'intervention qui, d'ailleurs, provoque son retour dans le 
cadre dialogique. Dans notre situation, ce sera le deuxième temps dans le destin de l'affect. En effet, seule la réactualisation de l'affect passif rend possible un éventuel développement du mouvement affectif à l'œuvre pour que du nouveau puisse éventuellement advenir. Mais à une condition: celle de prendre en charge, dans un cadre inhabituel pour cette répétition, cet évènement au cours duquel la lumière rallumée éteint une nouvelle fois le dialogue. En effet, cette répétition est cette fois adressée au dispositif d'intervention et à ceux qui le composent: opérateurs, direction entreprise, syndicats, intervenants-chercheurs. Dès lors, la lumière vite rétablie au poste, au prix de repousser dans l'ombre les empêchements dialogiques de l'organisation, peut devenir un objet d'analyse partagée si, au plan clinique, l'occurrence de l'événement est saisie et peut devenir l'instrument d'une requalification dialogique de la part des intervenants ou de tout autre protagoniste du Comité de suivi. Faute de quoi le cours même de l'intervention peut venir épouser et répéter les impasses d'un développement factice de l'activité de Direction. Dans le cas présent, il se révéla possible de transformer ce risque en atout grâce à la reprise, dans le cadre dialogique de la réunion du Comité suivant, de la déliaison potentielle entre la question de la lumière et celle de la "parole inutile » des opérateurs; en proposant de regarder ensemble cette répétition comme prototypique d'un rapport au dialogue "ravalé " avec les opérateurs sur le travail réel dans l'entreprise. Désindexer les questions techniques, soulevées par les analyses de l'activité dans le cadre du dispositif, des questions du dialogue organisationnel empêché conduit inévitablement à aggraver les inhibitions dialogiques. Le paradoxal activisme passif de la hiérarchie, discuté collectivement dans ce cadre, a permis la liaison entre dimension technique et dialogique des problèmes, et ainsi produit le développement d'un diagnostic commun sur la "parole inutile" des opérateurs. C'est l'instauration d'habitudes de dialogue pour trouver des solutions pour tous, d'abord « impensables », qui est visée ici, et non pas de simples colmatages techniques réalisés dans l'urgence. Mais ces habitudes renouvelées ne résultent pas d'un conseil en «bonnes pratiques ». Il s'agit plutôt de tirer le meilleur parti possible de la puissante vitalité des affects passifs initiaux de la Direction, par une prise en charge clinique qui pousse leur répétition au-delà de la répétition. La qualité du dialogue des opérateurs entre eux -que le film d'autoconfrontation donne à voir - autorise, au sens fort du terme (Bonnemain, PrunierPoulmaire, \& Clot, à paraitre) - un travail dialogique de «révision » des fonctions de la «lumière». Quoi qu'il en soit, cette bifurcation ouvrira une nouvelle phase de l'intervention: l'expérimentation dans l'atelier de temps institués de dialogue entre l'encadrement et les opérateurs pour découvrir et instruire les problèmes de travail insoupçonnés.

\section{Répétition : le devenir actif, temps trois}

Dans cette nouvelle phase, deux mois plus tard, le mouvement affectif engagé trouvera à se répéter une troisième fois, signant le devenir actif de ces affects passifs. Ce devenir n'était pourtant pas, encore une fois, écrit d'avance. Cette fois c'est à l'atelier, et non plus en Comité de pilotage, que la situation se noue, l'atelier où nous sommes alors occupés à expérimenter le dialogue entre encadrement et opérateurs : trois postes ont été choisis par les opérateurs en accord avec l'encadrement car ils condensent beaucoup de questions professionnelles non résolues. Nous mobilisons à nouveau la méthode des 
autoconfrontations croisées, mais, à ce stade, le montage vidéo sert d'instrument dialogique entre les opérateurs, l'encadrement et nous.

Le premier poste choisi pour l'analyse est celui dit des « lécheurs ». Du nom de la pièce de type joint en caoutchouc en forme de baguette qui «lèche» la vitre des portes du véhicule lorsque cette vitre entre et sort du caisson. Chaque côté de la vitre possède son " lécheur ». La tâche de l'opérateur consiste à le poser horizontalement sur la feuillure métallique du haut du caisson de porte et à le « clipser ». Dans le cas présent, cette pièce est affectée d'un défaut de conception récurrent. L'opérateur doit consentir des efforts anormalement élevés pour parvenir à monter la pièce et l'analyse montrera que ces efforts sont trois fois supérieurs aux normes ergonomiques tolérées, sachant, de plus, que chaque opérateur réalise cette tâche environ 300 fois par jour. Les premiers échanges rendus possibles par notre intervention autour de ce poste ont conduit le chef d'atelier à reconnaitre d'abord le problème technique puis à décider d'une solution palliative: la lubrification de la pièce avant son montage. Cependant, cette solution technique fait surgir une question imprévue. En effet, il faut affecter l'opération de lubrification à un autre opérateur car le titulaire du poste ne peut la réaliser en sus de son engagement. Son poste deviendrait «intenable» dans le temps de cycle alloué. Formellement le chef d'atelier demandera donc aux deux chefs d'équipe d'imputer la nouvelle tâche à l'opérateur sénior (OPS) ${ }^{1}$. En pratique, seul l'OPS de l'équipe A remplit effectivement cette tâche. Dans l'équipe $B$ c'est très aléatoire : les deux opérateurs concernés ont dû prendre l'habitude de s'affranchir de cette contribution extérieure, et nourrissent à l'égard de l'encadrement méfiance et ressentiment. Précisons que dans cette équipe les opérateurs sont majoritairement intérimaires et que le management y est plus péremptoire de sorte qu'il leur est très difficile de faire autorité dans leur travail (Bonnemain, PrunierPoulmaire, \& Clot, à paraître).

Lors de l'autoconfrontation croisée, cette différence sera découverte par l'opérateur intérimaire de l'équipe B. C'est avec surprise qu'il réalise alors que dans l'équipe A, pour son collègue titulaire depuis 10 ans, le problème de la lubrification est effectivement traité. Pour ce dernier, si la pièce n'est pas lubrifiée, c'est l'OPS qui s'en charge, et le cas échéant il peut laisser " passer » la porte afin qu'elle soit retouchée plus bas ou que la chaine soit arrêtée. Les affects éprouvés ici par l'opérateur comprenant que son collègue parvient malgré tout à peser sur cette situation de travail dégradée ne sont pas restés sans effet. Après l'autoconfrontation croisée, à son retour au poste, il refusera de monter les pièces non lubrifiées. Et c'est alors la colère qui emportera la réaction du Chef d'Unité (CU). Il considèrera la nouvelle posture de l'opérateur intérimaire placé sous sa responsabilité comme une conséquence inacceptable du travail conduit en autoconfrontation, transférant ainsi résistance et agressivité sur l'intervenant présent et, plus généralement, sur le dispositif d'intervention. Ici, l'intensité affective de l'activité du chef d'équipe est passivement polarisée. L'inattendu impose une révision douloureuse des équilibres artificiels d'une organisation du travail différente pour les deux équipes. Une rupture est introduite par le dispositif d'intervention dans les normes de pénibilité admises, incorporées même par l'opérateur et imposées, de fait, par un rapport hiérarchique marqué du sceau de la subordination intérimaire.

41 Cet événement conduira les intervenants à changer de destinataire et à retourner la question vers le registre impersonnel du travail, incarné par le Directeur d'usine : ces tensions sont «normales" au vu de l'imbrication très serrée entre un problème de conception de la pièce mal réglé et les attendus génériques de l'activité hiérarchique : un 
Chef d'Unité doit «tenir ses hommes ». Ces tensions sont "normales », mais ne doivent pas dégénérer au moment où s'expérimente le dialogue prévu après le Comité de pilotage. Confronté à l'embarrassante question des impasses du dialogue dans l'atelier, à la difficulté de sa "révision » chez les Chefs d'Unité, le Directeur d'Usine suggère tout de suite de "prendre sur lui » la recherche d'une solution au problème technique : sur un sujet de ce type c'est spontanément dans cette direction qu'il s'oriente. En effet, il ne dispose pas des marges de manœuvre financières pour pallier au problème en affectant un opérateur supplémentaire à la tâche d'enduire de glycol le lécheur mal conçu. À nouveau il semble donc se diriger vers ce qui ne peut être qu'un colmatage provisoire du conflit de critères qu'on lui soumet, vers une répétition de la recherche d'une solution technique immédiate. Encore une fois, c'est d'abord la réponse technique qui s'impose spontanément sans lien avec la question dialogique soulevée, réactivant la situation déjà vécue à propos de la «lumière" sur l'autre poste. Le Directeur parait s'absorber à nouveau dans cette « joyeuse passivité » de l'activisme technique. Il semble s'y oublier.

Pourtant, il se ravise alors et retourne vers les intervenants la question du dialogue. N'at-il pas justement «appris » avec eux, au cours du Comité de suivi précédent, que l'activisme technique, s'il peut bien éteindre à court terme les conflits interpersonnels ruine simultanément les ressources psychologiques et sociales du dialogue à expérimenter dans l'organisation? L'humour libère souvent l'énergie stérilisée des affects, ce que confirme notre situation: en réalisant sur l'instant, devant les intervenants, qu'il est de nouveau emporté par son mouvement "technique», le Directeur de l'usine fait une expérience : c'est l'humour et le rire partagés qui s'emparent alors de l'échange avec eux. Et le rire, «déblaye la voie ", pour le dire à la manière de Bakhtine (1970; Werthe, 2001), révélant, au passage, comment affects et émotions sont liés (Clot, 2016; Clot, sous presse) ${ }^{2}$. Un véritable "allègement " s'ensuit pour lui qui, d'abord interpellé par les intervenants se découvre en situation de s'enquérir auprès d'eux de la conduite à suivre. Il sera alors convenu que la question technique du «lécheur» mérite au plus haut point d'être travaillée, mais qu'elle doit, cette fois, devenir l'instrument d'un autre genre de rapports dialogiques dans les équipes concernées par la tâche en question. Deux actions s'en suivent, mais, par des «moyens détournés ", elles se prémunissent contre l'accélération factice et les solutions apparentes. Preuve qu'un renoncement à « faire comme d'habitude » n'est pas forcément marqué du signe de la passivité. Au contraire. La première action consiste à réaffirmer, auprès de l'encadrement de l'atelier, en prenant soin de ne pas le remettre en cause, le soutien de la Direction d'usine à l'engagement de chaque niveau hiérarchique dans le dialogue autour des dilemmes du travail réel. Et ce, en utilisant le dispositif méthodologique de l'intervention afin d'expérimenter d'autres façons de faire. La première réunion entre opérateurs et encadrement à propos du poste controversé aura bien lieu en restaurant la légitimité du dispositif d'intervention. Sans aucune naïveté quant à la « rugosité » des rapports sociaux, les différents problèmes et solutions y seront discutés, l'opération lubrification du lécheur sera bien engagée au poste (l'opérateur aura le temps de lubrifier la pièce) au moment même où l'encadrement pourra se prévaloir de l'engagement de la direction d'usine dans la re-conception de la pièce. Et c'est sur ce point que nous voudrions insister.

Car l'essentiel, à ce moment-là, est peut-être du côté d'une transformation des destinataires dans l'activité du Directeur d'Usine. En parallèle avec la restauration du dialogisme de l'expérimentation, il va se mesurer au problème technique de façon 
renouvelée en tournant le dos aux habitudes. La question du lécheur va devenir pour lui, à l'aide de l'intervention en cours, un moyen de questionner l'Entreprise au-delà de l'usine, pour redéfinir, sur l'objet même du lécheur, les rapports entre l'ingénierie de conception et la fabrication dans l'Usine. Pendant plusieurs semaines, et régulièrement une fois par semaine, il fera un "point d'avancée » sur l'analyse du problème en bord de chaine - sous le regard des opérateurs en poste mesurant ainsi sa prise en charge organisationnelle - avec le sous-traitant fabricant de la pièce, les « fonctions support » et l'ingénierie. Plusieurs mois plus tard, la pièce sera effectivement reconçue.

À propos de l'activité de direction, on peut certainement parler ici de devenir actif des affects dans l'action. La «révision» effective des organisateurs sociaux habituels de l'activité - que nous avons pris comme critère dans la définition des affects actifs - est bien au rendez-vous. Les forces centripètes du déjà-vécu deviennent un moyen de vivre autre chose, provoquant le développement effectif des forces centrifuges de l'action dans l'entreprise. C'est un pouvoir d'agir nouveau sur l'organisation qui s'expérimente au-delà même de l'Usine alors même que le protocole des habitudes bien rôdées aurait pu s'enliser dans les délais conventionnels propres à la «remontée des problèmes " classique. Les questions y sont rarement résolues. Ici, c'est l'inverse qui se produit. C'est l'organisation qui « descend » sur les problèmes pour les résoudre. Notons, pour finir, que le développement du dialogue dans l'usine et dans l'entreprise sur cette pièce du lécheur, et finalement son efficacité technique contribuera, en retour, au développement de l'expérimentation.

\section{Discussion}

À ce point nous pouvons identifier un paradoxe : le développement réalisé de l'activité ne se mesure pas unilatéralement aux vertus de l'acte effectif accompli. Ce dernier peut se révéler passif comme nous l'avons vu avec le traitement précipité du problème de lumière au poste, pourtant bien réel, provoqué par les affects en Comité de suivi. Nous avons vu qu'il est passif car il répète un fonctionnement à l'identique où l'épreuve affective sert paradoxalement de preuve pour valider les fonctionnements déjà installés ; où l'expérience qu'on fait n'est jamais que le moyen de confirmer l'expérience qu'on $a$. Les chemins vers le développement effectif de l'activité réalisée suivent des oscillations affectives au destin difficilement prédictible. Ils peuvent déboucher sur l'impasse d'actes accomplis qui sont pourtant autant d'obstacles au développement réel. C'est la répétition sans répétition (Bernstein, 1996) dans le cadre dialogique qui peut donner aux affects passifs un devenir actif dans l'action. Cette issue «finalement active " passe, en tout cas, par la prise en charge développementale de l'affect passif. C'est une condition nécessaire. Mais elle n'est pas suffisante (Bonnemain, 2015). Laissé à lui-même, l'affect passif peut empoisonner l'activité jusqu'à la faire dégénérer. L'intervention peut, au contraire, en alimentant le mouvement des affects, le régénérer en le « rechargeant » de son énergie dialogique pour étendre le pouvoir d'agir, ce rayonnement de l'activité (Clot \& Simonet, 2015). C'est le cas, comme dans notre exemple, quand l'action "retenue " transforme une abdication apparente, à l'allure pourtant "passive», en devenir actif effectif. On le voit, l'affect n'est pas seulement un éprouvé subjectif, mais une force vitale au destin imprédictible, ce qui ne veut pas dire inexplicable. Source d'énergie pour l'activité, il trouve dans cette activité de quoi se développer selon des voies qui peuvent varier en fonction de l'intensité des échanges dialogiques en cours d'action. L'affect se 
développe quand l'affectivité est mise en activité. Le développement du pouvoir d'agir est même à ce prix.

On mesure alors la portée de la perspective développementale proposée par Vygotski : si l'affectivité elle-même peut se développer en cours d'activité, on comprend qu'il écarte toute tentation d'investir l'affectivité d'un pouvoir générateur originaire. Notre exemple montre qu'elle est nomade. Elle ne tient pas en place. C'est que l'affectivité ne vit pas seule, déliée de tout le reste de la vie psychique en rapport avec l'activité. Elle ne reste pas inchangée ou égale à elle-même tout au long du développement de cette même activité. Vygotski insiste : pas plus que « l'intellect ne peut s'expliquer complètement par lui-même» (1994, p. 218), l'affectivité ne saurait être comprise seulement du dedans, comme "un élément original, propre et autonome, isolé des conditions réelles d'existence (...) comme un fait qui ne peut être modifié au cours du développement » (Ibid p. 219). Elle dépend du premier conflit triadique de l'activité qui retentit sur le second au cours duquel le "déjà vécu » est révisé, plus ou moins brutalement. L'énergie ainsi libérée nous relie aux situations réelles (Jouanneaux, 2011), mais elle suit des chemins différents et donne à la vie des « allures » plus ou moins opposées et accentuées selon les cas (Canguilhem, 1984, p. 137).

Au bout du compte l'activité peut se régénérer comme notre exemple le montre, toujours au risque de dégénérer. L'intervention et l'activité du clinicien n'en sont que plus importantes (Scheller, 2013). G. Deleuze peut nous aider à formuler le problème encore autrement: «les affects ne sont pas des sentiments, ce sont des devenirs qui débordent celui qui passe par eux (il devient autre) » (2003, p. 187). On sait que Vygotski voyait dans le développement de l'activité et ses empêchements fréquents la preuve des idées de l' Éthique de Spinoza (1965) «qui entend par affects d'une part ces états corporels qui augmentent ou diminuent l'aptitude du corps lui-même à l'action, la favorisent ou la restreignent, et d'autre part les idées que l'on a de ces états " (Vygotski, 1998, p. 105). Il semble bien, si l'on en croit les trois temps de la répétition propres à la situation exposée ci-dessus, qu'il ait eu raison d'explorer cette piste développementale même pour l'affectivité. L'affect nous renvoie en fait immédiatement à une façon de vivre ou transforme cette façon de vivre. Pour Spinoza être affecté c'est effectivement se mettre à faire quelque chose d'une certaine manière, être disposé de telle ou telle manière (Bove, 2009, p. 104).

\section{Le développement?}

Mais il est vrai qu'il faut alors se faire une idée précise du développement. On conclura donc sur ce point. Dans le cadre méthodologique mobilisé ici, le développement du sujet, du collectif ou encore de l'organisation prescrite n'est pas une trajectoire dont le but est connu d'avance. Son modèle n'est pas embryologique. Il ne va pas vers sa fin en réalisant sa nature initiale, sa potentialité intrinsèque. Il suffirait alors seulement d'affranchir cette puissance potentielle des empêchements inopportuns du réel qui la dévie de sa trajectoire normale. Il suffirait de "libérer» de l'extérieur les potentialités d'une puissance d'agir déjà là. Le développement dont il est question ici, dans une perspective qui emprunte aussi bien à Vygotski $(1994$; 2014) qu'à Dewey, fait surgir des formes nouvelles dans l'expérience, qui la régénère et l'empêche de mourir d'inanition (Dewey, 2010, p. 114). Là où, en cours d'action, il y a changement, écrit Dewey, « il y a instabilité, et l'instabilité est preuve que quelque chose se passe, qu'il y a de l'absence, du manque, de 
l'incomplétude » (Dewey, 2014, p. 164). Dans ce cadre clinique l'intervention ne peut pas être, pour reprendre une formule de J. Bruner, "un amortisseur contre l'inattendu " (2014, p. 44) car c'est l'orientation dominante de l'affect - entre passivité et activité qui décide de sa trajectoire de transformation. Elle est toujours aux prises avec les forces centripètes et centrifuges qui tirent la "révision » de l'expérience dans des directions opposées. Au bout du compte on comprend que, de ce point de vue, le développement soit à la fois l'objet et la méthode de l'intervention en clinique de l'activité. On comprend aussi qu'une ergonomie constructive puisse retrouver cette perspective :

«L'ergonome ne peut pas afficher le développement comme objectif de la discipline sans se faire l'avocat de méthodologies d'intervention qui elles-mêmes favorisent le développement. La participation active des opérateurs aux démarches de changement et de conception n'est pas une caractéristique "additionnelle", "optionnelle" de la méthodologie de l'action ergonomique. Elle est nécessaire pour assurer la cohérence d'une démarche constructive » (Falzon, 2013, p. 4). ses destins.

\section{BIBLIOGRAPHIE}

Almudever, B., Michaëlis, N., Aeschlimann, M. P., \& Cazals-Ferré, M. P. (2011). Le pouvoir d'agir à l'épreuve de la souffrance au travail : émotions, recherche et construction de sens. Psychologie du travail et des organisations, 18(1), 52-64.

Arnoud, J., \& Falzon, P. (2013). La co-analyse constructive des pratiques. In P. Falzon (Ed.), Ergonomie Constructive, (pp. 223-236). Paris : PUF.

Bakhtine, M. (1970). L'œuvre de François Rabelais et la culture populaire au moyen âge et sous la renaissance. Paris : Gallimard.

Bautier, E., \& Rochex, J. Y. (1999). Henri Wallon. L'enfant et ses milieux. Paris : Hachette.

Bernard, J. (2015). Les voies d'approche des émotions. Enjeux de définition et catégorisations. Terrains/Théories [en ligne], 2.

Bernstein, N.A. (1996). On dexterity and its development. In M.L. Latash, \& M.T. Turvey (Eds.), Dexterity and its development. New Jersey : LEA.

Bonnefond, J. Y. (2015). Une expérience d'amélioration de la qualité du travail à Renault-Flins. La revue des conditions de travail, ANACT, 3, 66-74.

Bonnemain, A. (2015). Les paradoxes de l'intensité affective en autoconfrontation. Dialogues chez des chefs d'équipe de la propreté de Paris. Thèse pour le Doctorat en psychologie. Paris : CNAM.

Bonnemain, A., \& Clot, Y. (sous presse). Clinique de l'activité : les affects dans l'autoconfrontation. In M. Santiago-Delefosse, \& M. del Rio Carral (Eds.), Les méthodes qualitatives en psychologie. Paris : Dunod.

Bonnemain, A., Prunier-Poulemaire, S., \& Clot, Y. (sous presse). Faire autorité dans le métier ? Du collectif au Comité de Pilotage. Un exemple dans une grande collectivité. Pistes. 
Bove, L. (2009). Émotions, manières d'être et nature humaine chez Spinoza. In S. Roux (Ed.), Les émotions. Paris : Vrin.

Briec, C. (2013). Syndicalisme : « l'impersonnel » à l'épreuve. Le cas d'une section départementale $\mathrm{du}$ SNUIPP-FSU. Thèse pour le doctorat de psychologie. Paris : CNAM.

Bruner, J. (2014). Culture et esprit : une féconde incommensurabilité. In de C. Moro \& N. Muller Mirza (Eds.), Sémiotique, culture et développement psychologique. Villeneuve d'Ascq : Septentrion.

Cahour, B. (2010). Émotions, affects et confort comme nouveaux déterminants de l'activité et de l'usage. In G. Valléry, M-C. Le Port, \& M. Zouinar (Eds.), Ergonomie, conception de produits et services médiatisés (pp. 273-305). Paris : PUF.

Cahour, B., \& Lancry, A. (2011). Émotions et activités professionnelles et quotidiennes. Le travail humain, 74(2), 97-106.

Cahour, B., \& Licoppe, C. (2010). Confrontations aux traces de son activité. Compréhension, développement et régulation de l'agir dans un monde de plus en plus réflexif. Revue d'anthropologie des connaissances, 4(2), 243-253.

Canguilhem, G. (1983). Études d'histoire et de philosophie des sciences. Paris : Vrin.

Canguilhem, G. (1984). Le normal et le pathologique. Paris : PUF.

Clot, Y. (1999). La fonction psychologique du travail. Paris : PUF.

Clot, Y. (2008). Travail et pouvoir d'agir, Paris : PUF.

Clot, Y. (2010). Le travail à cœur, pour en finir avec les risques psychosociaux, Paris : La Découverte.

Clot, Y. (2013). Suicides au travail. Un drame de la conscience professionnelle ? Activités, 10(2). https://activites.revues.org/716.

Clot, Y. (2014). Clinique de l'activité. In P. Zawieja, \& F. Guarnieri (Eds.). Dictionnaire des risques psychosociaux. Paris : Le Seuil.

Clot, Y. (2015a). Les affects et l'action. Préface à la 6ème édition. In Y. Clot, La fonction psychologique du travail. Paris : PUF.

Clot, Y. (2015b). Vygotski avec Spinoza, au-delà de Freud. Revue philosophique, 2, 205-223.

Clot, Y. (2016). Activité, affect : sources et ressources du rapport social. In M.-A Dujarier,

C. Gaudart, A. Gillet, \& P. Lénel (Eds.), L'activité en théories - Regards croisés sur le travail (pp. 76-93). Toulouse : Octarès.

Clot, Y. (sous presse). L'affectivité en activité. In J.M. Barbier, \& M. Durant (Eds.), Encyclopédie d'analyse des activités. Paris : PUF.

Clot, Y. \& Faïta, D. (2000). Genres et styles en analyses du travail : concepts et méthodes. Travailler, 4, 7-43.

Clot, Y., Faïta, D., Fernandez, G., \& Scheller, L. (2001). Entretiens en autoconfrontation croisée : Une méthode en clinique de l'activité. Éducation permanente, 146, 17-26.

Clot, Y., \& Gollac, M. (2014). Le travail peut-il devenir supportable. Paris : A. Colin.

Clot, Y. \& Simonet, P. (2015). Pouvoirs d'agir et marges de manœuvre. Le Travail Humain, 78(1), 37-49.

Dejours, C., \& Gernet, I. (2012). Psychopathologie du travail. Issy-les-Moulineaux : Elsevier Masson. 
Deleuze, G. (2003). Pourparlers. Paris : Minuit.

Dewey, J. (2010). L'art comme expérience. Paris : Folio.

Dewey, J. (2014). Reconstruction en philosophie. Paris : Folio.

Duboscq, J., \& Clot, Y. (2010). L'autoconfrontation croisée comme instrument d'action au travers du dialogue : objets, adresses et gestes renouvelés. Revue d'anthropologie des connaissances, 4(2), 255-286.

Falzon, P. (2013). Pour une ergonomie constructive. In P. Falzon (Ed.), Ergonomie Constructive. (pp. 1-15). Paris : PUF.

Faverge, J-M. (1979). Le travail en tant qu'activité de récupération. Bulletin de Psychologie, Tome XXXIII, 203-206.

Fernagu-Oudet, S. (2012). Concevoir des environnements de travail capacitants : l'exemple d'un réseau réciproque d'échanges des savoirs. Formation emploi, 119, 7-27.

Fernandez, G. (2009). Soigner le travail. Itinéraires d'un médecin du travail. Toulouse : Erès.

Green, A. (2004). Le discours vivant. Paris : PUF.

Hochschild, A.R. (2003). Travail émotionnel, règles de sentiments et structure sociale. Travailler, 9 (1), 19-49.

Jeantet, A. (2003). L'émotion prescrite au travail. Travailler, 9(1), 99-112.

Jouanneaux, M. (2011). De l'agir au travail. Toulouse : Octarès.

Kitanaka, J. (2014). De la mort volontaire au suicide au travail. Paris : Ithaque.

Kloetzer, L., Clot, Y. \& Quillerou-Grivot E. (2015). Stimulating Dialogue at Work: The Activity Clinic Approach to Learning and Development. In L. Fillietaz, \& S. Billet (Eds.). Francophone perspectives of learning through work - Conceptions, traditions and practices (pp. 49-70). Springer.

Kostulski. K. (2011). Formes et fonctions psychologiques des réalisations langagières : vers une psychologie concrète du langage. Thèse d'habilitation à diriger des recherches, Université Paris 8.

Kostulski, K. (2012). La diversité fonctionnelle du langage : usages et conflictualités dans l'activité. In Y. Clot (Ed.). Vygotski maintenant (pp. 237-254). Paris : La Dispute.

Kostulski, K., \& Clot, Y. (2007). Interaction et migration fonctionnelle : un développement en autoconfrontation croisée. In Y. Clot \& K. Kostulski (Eds.), Dialogue, activité, développement, Psychologie de l'interaction, 23-24, 73-109.

Kostulski, K., Clot, Y., Litim, M., \& Plateau, S. (2011). L'horizon incertain de la transformation en clinique de l'activité : une intervention dans le champ de l'éducation surveillée. Activités, 8(1), 129-145. https://activites.revues.org/2456.

Livet, P. (2002). Émotions et rationalité morale. Paris : PUF.

Lhuilier, D. (Ed.) (2014). Qualité du travail, qualité au travail. Toulouse : Octarès.

Litim, M. (2012). Les méthodes indirectes à l'épreuve de la pratique : questions d'intervention. In Y. Clot (Ed.) Vygotski maintenant. Paris : La Dispute.

Livet, P. (2007). Émotions et révision : la dynamique des débats. In C. Blatrix et al., (Eds.), Le débat public : une expérience française de démocratie participative (pp. 339-352). Paris : La Découverte. 
Macherey, P. (1999). Descartes et Spinoza devant le problème de l'usage des passions. In C. Lazzeri (Ed.), Spinoza, puissance et impuissance de la raison (pp. 93-114). Paris : PUF.

Miossec, Y., Clot, Y., \& Boucher, C. (2014). L'intervention dialogique pour agir sur les risques psychosociaux : une troisième voie. In L. Lerouge (Ed.), Approche interdisciplinaire des risques psychosociaux au travail (pp. 51-67). Toulouse : Octarès.

Molinier, P. (2009). De la rumeur à la peur. Une enquête de psychodynamique du travail à la suite d'un suicide lié au travail. Communication et organisation, 36, 44-54.

Pastré, P. (2012). La didactique professionnelle. Approche anthropologique du développement chez les adultes. Paris : PUF.

Phillips, A. (2005). La mort qui fait aimer la vie. Darwin et Freud. Paris : Payot.

Poussin, N. (2014). Développement des sentiments au travail : dialogues sur l'efficacité et l'utilité chez des médecins du travail. Thèse pour le doctorat de psychologie. Paris : CNAM.

Poussin, N. (2015). Développement du sentiment d'efficacité : un développement du rapport entre affectivité et pensée. Activités, 12(1), 89-103. https://activites.revues.org/1028.

Poussin, N. \& Miossec, Y. (2015). Développement du sentiment et du pouvoir d'agir : un exemple chez des médecins du travail. Le Travail Humain, 78(1), 81-96.

Prot, B. (2006). La fonction du clinicien dans les méthodes de clinique de l'activité. Nouvelle revue de psychosociologie, 1(1). 31-44.

Quillerou-Grivot, E., \& Clot, Y. (2014). La qualité du travail : un problème avant d'être une solution. In D. Lhuilier (Ed.), Qualité du travail, qualité au travail. (pp. 141-159). Toulouse : Octarès.

Reille-Baudrin, E., \& Zittoun, M. (2015). Meurtre de deux inspecteurs du travail dans l'exercice de leur métier : re-trouver l'événement pour sortir de l'angoisse. Nouvelle revue de psychosociologie, 1 (19). 65-78.

Rimé, B. (2009). Le partage social des émotions. Paris : PUF.

Rochex, J. Y. (2002). Vygotski et Wallon : pour une pensée dialectique des rapports entre pensée et affect. In Y. Clot (Ed.). Avec Vygotski (pp. 121-139). Paris : La Dispute.

Roger, J. L. (2007). Refaire son métier. Essai de clinique de l'activité. Toulouse : Erès.

Ribert-Van de Weerdt, C. (2011). Les contraintes de travail et les stratégies de régulation émotionnelle en centre de relation clientèle. Le Travail Humain, 74(4), 321-339.

Scheller, L. (2013). Travail, affects, activité transférentielle. Activités, 10(2), 249-260. https:// activites.revues.org/853.

Severac, P. (2005). Le devenir actif chez Spinoza. Paris : Honoré Champion.

Tomàs, J.L., \& Bonnefond, J.Y. (2014). De « l'épuisement professionnel » à la qualité du travail : le cas de la « belle table d'instrumentation ». In D. Lhuilier (Ed.), Qualité du travail, qualité au travail. (pp. 129-140). Toulouse : Octarès.

Spinoza, B. (1965). Éthique. Première édition 1677. Paris : Flammarion.

Van Belleghem, L., De Gasparo, S., \& Gaillard, I. (2013). Le développement de la dimension psychosociale au travail. In P. Falzon, (Ed.), Ergonomie Constructive. (pp. 47-60). Paris : PUF.

Veil, C. (2012). Vulnérabilités au travail. Toulouse : Erès.

Vygotski, L. (1994). Défectologie et déficience mentale. Neuchatel : Delachaux et Nieslé. 
Vygotski, L. (1997). Pensée et Langage. Paris : La dispute.

Vygostki, L. (1998). Théorie des émotions. Paris : L’Harmattan.

Vygotski, L. (2003). Conscience, inconscient, émotions. Paris : La Dispute.

Vygotski, L. (2014). Histoire du développement des fonctions psychiques supérieures. Paris : La Dispute.

Wallon, H. (1982). La vie mentale. Paris : Éditions sociales.

Werthe, C. (2001). Le rire et ses ressources en clinique du travail. Éducation permanente, 146, 193-203.

\section{NOTES}

1. Opérateur extérieur à la chaine et qui intervient, à la demande, sur les problèmes d'approvisionnement, sur les retouches, et sur les outils

2. Vygotski a approché ces processus à l'aide de la notion de Perezhivanie (Clot, 2016).

\section{RÉSUMÉS}

Cet article étudie le rapport entre affectivité et activité dans les interventions en psychologie du travail, en l'occurrence sous les modalités de la clinique de l'activité. L'affect, que nous distinguons des émotions, est défini comme un conflit de l'activité avec elle-même, un rapport de force qui met en balance les attendus et les inattendus de l'activité, dont le devenir - imprédictible - oscille entre deux orientations, plutôt active ou passive. À partir d'un exemple tiré d'une intervention dans l'industrie automobile, nous montrons que l'affectivité est mise au travail dans l'intervention et qu'en se développant, passivité et activité peuvent s'inverser, l'une peut prendre le visage de l'autre. En effet, le cadre clinique dialogique est la source du développement possible de l'affect pour un devenir actif à une condition: quand l'affect fait l'objet d'une reprise dans un autre contexte où sa répétition est non seulement admise, mais sollicitée par l'intervention. Celle-ci peut alors, en alimentant le mouvement des affects, le régénérer en le « rechargeant » de son énergie dialogique pour étendre le pouvoir d'agir.

This paper examines the relationship between affectivity and activity during psychology interventions at work, particularly in terms of clinical activity. Different from emotion, affect is defined as a conflict within the activity, as a balance of power between what is expected and unexpected in the activity, the unpredictable future of which oscillates between two orientations, either more active or more passive. Based on an example from an intervention in the automotive industry, we show that the intervention causes movements in affectivity. During these movements, activity and passivity can be reversed, and one might eventually mutate into the other. Indeed, the dialogic clinical setting can be the source of a potential development of the affect, leading to an active orientation towards one condition: when the affect is the subject of repetition in another context, where repetition is not only permissible but specifically solicited 
by the intervention. In causing the affective movement, the intervention might regenerate it by "reloading" it with its own dialogical

INDEX

Keywords : affect, activity, intervention, development, power to act

Mots-clés : affect, activité, intervention, développement, pouvoir d'agir

\section{AUTEURS}

\section{JEAN-YVES BONNEFOND}

CNAM, Équipe Psychologie du travail et clinique de l'activité, CRTD, 41, rue Gay-Lussac, 75005 Paris - jeanyves.bonnefond@cnam.fr

\section{YVES CLOT}

CNAM, Équipe Psychologie du travail et clinique de l'activité, CRTD, 41, rue Gay-Lussac, 75005 Paris - yves.clot@cnam.fr 